\title{
A Cross layer QoS Framework for Heterogeneous IoT environment
}

\author{
Ravi C Bhaddurgatte, Vijaya Kumar B P, Kusuma S M
}

\begin{abstract}
Internet of Things (IoT) is one of the fast growing technological paradigm in terms of architecture, standards, protocols, infrastructure deployment, Quality of Service (QoS), Service Level Agreements (SLAs), service provisioning, cross domain and cross platform implementations. IoT involves the techniques and technologies for sensing, actuation, communication, computation, networking and storage. In such a demanding environment the need for cross layer QoS functionalities are essential to address the issues like resources, mobility, security and energy management. The detailed review of literatures on IoT architectures and QoS implementations is made and it is observed that there is a need for cross layer QoS model in IoT environments and is one of the critical research challenges. A novel approach to address the above challenge(s) in an IoT environment requires an appropriate lathering of functional modules to different layers to meet different QoS requirements. Hence we propose a novel cross layer QoS framework supporting adaptable and distributed decision making in the IoT environment as a cross layer implementation addressing energy optimization and bandwidth efficiency. The results are verified by implementing the proposed framework in realistic IoT systems for verifying QoS parameters like delay, energy and bandwidth.
\end{abstract}

Keywords : Internet of Things [IoT], Quality of Service [QoS], Service Oriented Architecture [SOA], Heterogeneous network [hetnet], Service Level Agreement [SLA]

\section{INTRODUCTION}

IoT considers and includes the functionalities of information gathering, information processing, analysis, networking and communication for the overall IoT paradigm [1], [2]. An IoT system is an extension to existing heterogeneous network systems with additional and extended nodes such as Radio Frequency Identification (RFIDs), Sensors, Actuators, machines, smart mobiles and other identifiable objects / applications on Internet and Global Position System (GPS). These extended nodes get the real world data to the Internet and onto the systems for different applications by systems and end users. IoT with increased sophistication and ubiquitous nature [1] has many open

Revised Manuscript Received on December 30, 2019.

* Correspondence Author

Ravi C. Bhaddurgatte, Research Scholar, Computer Science and Engineering Department, Jain University, Bangalore, India. bcravi@yahoo.com.

Dr. Vijaya Kumar B. P., ISE Department, M S Ramaiah Institute of Engineering and Technology, Bangalore, India. vijaykbp@yahoo.co.in.

Kusuma S. M., Telecommunication Engineering Department, M S Ramaiah Institute of Engineering and Technology, Bangalore, India. kusumasm@msrit.edu.

(C) The Authors. Published by Blue Eyes Intelligence Engineering and Sciences Publication (BEIESP). This is an open access article under the CC BY-NC-ND license (http://creativecommons.org/licenses/by-nc-nd/4.0/) research areas to be addressed as discussed by John [1] \& as in [2], [10] on various enabling technologies and application areas. QoS issues and challenges in heterogeneous networks and IoT are being discussed in various research papers at various research levels. QoS issues in IoT still require more research in terms of analysis, design, modeling, protocols, and standardization, architecture, security and QoS solutions. Compared to QoS in traditional networks, the QoS in IoT environment is relatively less stabilized. More research and improvement is needed for QoS issues in IoT as the paradigm of IoT is one of the fast growing one, and because of the scalability, wide spread application areas, deployment complexities and ubiquitous nature. The traditional QoS parameters are inappropriate for IoT environment [3]. It is crucial to bring in multi-objective optimization [2] for effective QoS implementation, decision making and adaptability to deal with critical QoS parameters like energy, information accuracy, security, throughput, availability, response time and bandwidth utilizations.

In the proposed work, QoS is implemented and managed by a cross layer QoS framework to guarantee the total QoS by adaptability and decision making. The model uses the IoT functional modules as re-distributable across layers to lather the functional modules to appropriate layer for achieving effective QoS. Implementations are carried out to measure the operational efficiency with respect to energy usage and bandwidth using distributed decision making. The result shows that the proposed framework has given an improved operational efficiency in IoT systems for energy and bandwidth.

The paper is organized into remaining sections as, the details the review of related research works in section II, and section III discusses the proposed QoS architecture and cross layer framework for IoT environment, the implementation and results are discussed in section IV, and finally the paper discusses the conclusion in section $\mathrm{V}$.

\section{LITERATURE REVIEW}

From the literature review it is determined that the QoS, QoS implementation and QoS support is embedded into the IoT systems at Individual layers by different methods like 'algorithms and techniques for scheduling and optimization' at perception layer for field nodes, and at network layer by 'scheduling and routing'. QoS is also implemented based on various concepts and research ideas like - 'QoS based on message classification', 'QoS aware service oriented IoT', 'Using priorities and grouping for QoS', 'behavior patterns of intelligent sensor applications for QoS', 'service models used for implementing QoS'. 


\section{A Cross layer QoS Framework for Heterogeneous IoT Environment}

The IoT-A (Internet of Things Architecture) reference model in [17] discusses the IoT domain areas which are very wide. There is no single architecture / model for all IoT implementations, so there a need for a reference model to arrive at specific model/architecture. The reference model concept for IoT architecture is one of the motivation to define a cross layer QoS framework. As per the vision by IoT-A on Internet of Things, the main motivation for creating the reference model is to bring in interoperability of solutions at the communication level, as well as at the service level across various platforms which defines a model with common understandings [17]. There are various conceptual and practical architectures for IoT for techno-functional and QoS implementations [2] [3] [6], and these architectures are using three layer architectures with layers as: perception layer, network layer and application layer. IoT-A defined an IoT reference model and IoT architectural reference model by IoT European Research Cluster (IERC) which can be used to arrive at IoT reference architecture. The process and steps which can be used to arrive at any specific architecture [17].

The authors in [3] explains the implementation of a QoS model in IoT architecture with top down decision making process across three layers (application, network and sensing) using different optimization algorithms at each layer. The implementation of message scheduling algorithms for IoT sub groups (Priority \& Effort based) by service differentiation and assigning them traffic priorities is implemented in [4] which works for cross layer QoS support using multihop-Low Energy Adaptive Clustering Hierarchy(LEACH) method [4].

The authors in [5] study different QoS implementations in IoT environment for intelligent sensors applications (mote) and provisions QoS improvement. The QoS implementation done by mapping the behavioral patterns to QoS parameters (reliability, availability, energy efficiency, accuracy and real-time) as a cross-layer solution and impacts behavior patterns of mote applications. The adaptability of QoS implementation across layers, should depend on the dynamic behavior patterns. The common behavior patterns are: one-shot vs. iterative, fixed period vs. changeable, schedulable vs. non-schedulable, Initiative vs. reactive [5]. QoS architecture in [6] is defined by the three layer architecture, and by classifying application types and QoS levels for implementing the QoS in IoT.

In [7] the QoS support is based on the concept of general publish/subscribe for service oriented \& general IoT systems. The QoS aware implementation for publish/subscribe system for IoT uses methods of 'deadline aware system' and 'smart dispatch' for implementing QoS mechanism in IoT systems. This was missing in traditional publish/subscribe systems, the message broker is modified in these systems to meet QoS in IoT systems and results in better resource allocation and minimizes number of failures [7]. An approach for QoS service of RFID tracking systems is discussed in [8]. This approach optimizes the number of packets exchanged between reader and tags by optimizing the collisions of responses between tags and reader is another energy efficiency QoS implementation using 'anti-collisions protocols'.

Authors in [9] describe the QoS enablement based on services models and service differentiation for WSNs integration in IoT which is achieved by the categorization of IoT applications and service models. For availability, response time and reliability as QoS metrics in wireless sensor network (WSN)/IoT a computational modeling based on QoS using routing protocols is presented which uses directed graph and mathematical models for different service compositions.

The QoS maximization and network lifetime improvement using a new consensus protocol with distributed optimization is presented in [14] by Giuseppe et al for a resource constrained IoT environment.

For utilizing the cloud services, IoT systems use the cloud as an extended system. Consumers and service providers will have opposite and conflicting preferences in terms of service level agreements (SLAs). For achieving the QoS in a cloud based IoT environment a negotiation protocol based on the 'game of chicken' is adapted for agreements of SLAs and QoS [18].

Extending the QoS for IoT applications using 3GPP LTE-advanced (LTE-A) networks is another QoS scheme in IoT. The 3GPP LTE-A systems have discontinuous reception/transmission (DRX/DTX) mechanism to maximize the sleep periods of devices and guarantee their QoS and energy saving. For achieving the optimization of DRX/DTX parameters to support QoS in IoT DRX/DTX is extended in an IoT environment by guaranteeing packet loss rate, packet delay and traffic bit-rate [12].

The multi-dimensional QoS for parameters energy, accuracy and latency in WSNs in a real pervasive healthcare fall-detection application is achieved by adding additional modules in [15]. For the locally calculated consensus locally, gets the global consensus in an iterative manner for achieving QoS improvement in a service oriented IoT. The QoS improvement is implemented by improving trustworthiness and robustness using a decision making process and distributed consensus algorithm [16].

The detailed review of QoS architectures and implementations in the IoT environments [19] indicate the usage of hardware/devices, protocols, middleware components, specific algorithms and combination of hardware and software components to achieve QoS. Some of the architectures design with additional layers are basically implemented by splitting the functions of these layers into more than three layers for ease of support, maintenance and implementation. The IoT environment is dynamic and ubiquitous, the need for adaptable algorithms for scheduling and routing and distribution of computing is a major research area to explore. Though there are various approaches and models to achieve QoS improvements, a comprehensive QoS model for these dynamic IoT environment with adaptability, computation distribution in combination with dynamic routing seem to be missing as per the understanding and investigations made by the authors.

In this work we propose a comprehensive cross layer QoS model for implementing the concepts of dynamic, adaptive, auto configurable functional components for achieving effective QoS for energy and bandwidth.

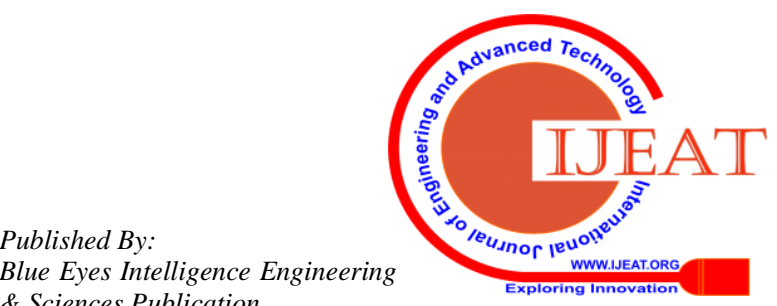




\section{PROPOSED ARCHITECTURE AND CROSS LAYER FRAMEWORK}

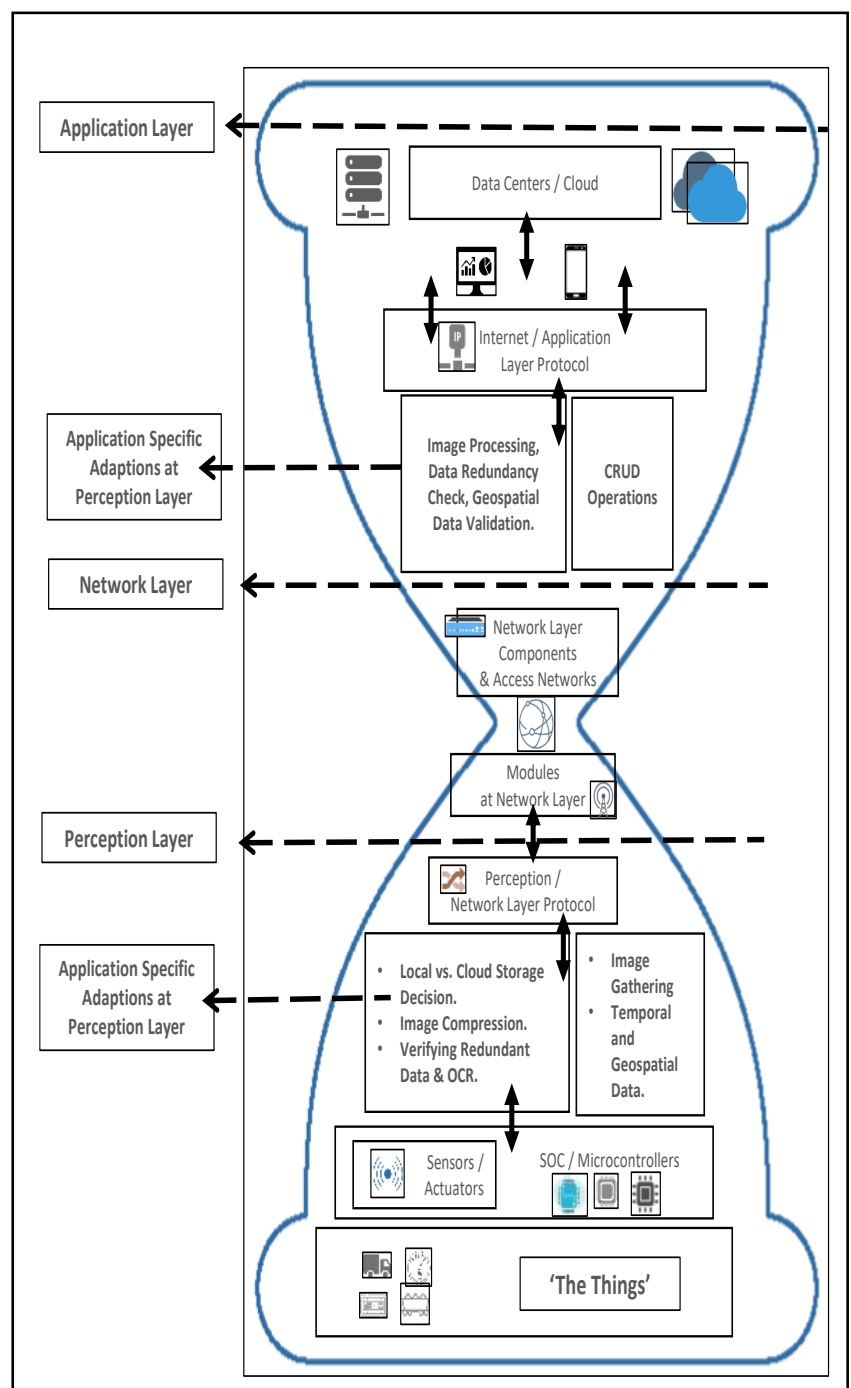

Fig. 1. Three Layer hourglass IoT architecture embedding the functional modules at each layer

There are loosely defined architectures, protocols, standards, and management system for IoT [1] [2] [10]. There are many defined conceptual architectures, services and service oriented architectures [16] discussed and implemented by individual researchers, and there are specific architectures adapted and implemented in product companies. Most of the IoT architectures are based on service oriented architecture concepts. The IERC provides IoT-A, the architecture reference model [17] for IoT which defines the reference model and detailed steps to arrive at specific architecture for any IoT implementations.

For our research work we adapt a three-layer architecture in the hourglass shape (narrow neck) as in Fig. 1, to indicate the limited control in network layer functions and large functional components at end layers. Managing and controlling the IoT functional modules can be majorly possible in application and perception layers, and in most of the IoT systems the major functions of IoT are implemented at two end layers.

The network layer does the layer specific functions (similar to mobile networks), as it requires some of the critical issues (ex. adaptability, dynamic IP addressing) to be tackled to get control and manage the deployable components/network devices which are majorly on public domain.

The functions of the three layer architecture are briefly discussed to identify the adaptable and distributable functions at the cross layer and for better understanding of the functions for QoS framework implementation.

Application Layer: The applications, systems and end-users of this layer consists of functional which consume the real world data. The real world action is taken based on the analysis, computations and decisions using the real world [19].

- $\quad$ End Users, Applications \& Systems: The functional modules in the application layer will be domain specific. The functions and actions by the functional modules in this layer will be based on the data received from lower layers representing the real objects or situations. These modules are not adaptable which are required at application layers. These modules are not adaptable which are required at application layers.

Modules specific to IoT data: As the IoT data is continuous and large requires the functions of optimizing for redundant and duplicate data from field devices. The functional modules also would include the functions of storing data for historic references, and data retrieval for dynamic decisions and adaptability and based on historic data. For effective QoS these modules and components can be considered as adaptable \& distributable between application \& perception layers.

Network Layer: The data from lower layer will be routed or communicated to upper layer and vice versa by the network layer. Network layer will have the components for networking and communication devices, protocols and routing / communication functions.

Perception Layer: The data sensing / gathering are the functional modules in the perception layer which collect the data from the machines, real world connected objects, and people. Using the combination of control commands received by upper layers and application systems / users of the domain and sensed data, the additional functional modules in the layer can perform the functions of controlling field devices / actions.

- $\quad$ Edge Nodes: The edge nodes in the IoT systems are basically the smart embedded devices such as System on Chips (SoCs), Microcontrollers etc. with limited of storage, computing and communication capabilities.

Field Devices: These are devices sensing the real world data with or with-out any intelligence and are capable of sensing information / data for a specific domain / application. For example sensors, RFID, actuator, mobile devices and other objects accessible directly or indirectly and providing the field data in the IoT environment by enabling IoT application for actions using real world data / situation. The layer components can include devices, machines and objects with computing capabilities and participating in IoT data. 


\section{A Cross layer QoS Framework for Heterogeneous IoT Environment}

The data gathering can happen either at perception layer from filed devices, if the layers are reversed upside down then the data sensing and gathering for IoT enablement can also happen at application layer if the data is from devices like smart phones or other Internet enabled smart machines.

Based on the review and study of existing IoT architectures and QoS implementations, the proposed cross layer QoS framework which categorizes the functionalities (with proper definitions given to different parameters) and their services to support the distributive nature of the IoT platform in a heterogeneous environment. The functional modules, architecture layers, QoS parameters and SLAs are tightly linked, interdependent, and related to each other. The model is based on the concept of adaptability and distribution / re-distribution of the IoT functions / functional modules on to any selected layer for achieving effective QoS and SLAs. SLAs which are always tied with quality of service and other contractual agreements between users and different service providers. The priorities of QoS requirements by stakeholders and architecture perspective could be different, but the implementation and management should be done by an integrated QoS model.

The QoS parameters are the factors that defines and control the quality of service (QoS). The QoS parameters for each layer are different and some of the QoS parameters will have impact on QoS across layers and they are considered as cross layer QoS parameters. The QoS parameters identified at high level for different IoT layers as per various reviews and implementations are summarized as listed in the review paper [19]. The Table 1 provides the layer wise and cross layer QoS parameters.

\section{TABLE 1 QoS Parameters and QoS Metrics}

\begin{tabular}{|c|c|c|}
\hline IoT Layer & $\begin{array}{l}\text { QoS Parameters and QoS } \\
\text { Metrics }\end{array}$ & $\begin{array}{l}\text { Cross Layer QoS } \\
\text { Parameters }\end{array}$ \\
\hline $\begin{array}{l}\text { Application } \\
\text { Layer }\end{array}$ & $\begin{array}{l}\text { - Service time, Availability of } \\
\text { service, } \\
\text { - Accuracy of service, Service } \\
\text { delay, Service load \& } \\
\text { priority } \\
\text { - Information or Data accuracy } \\
\text { - Network deployment cost, } \\
\text { Service usage cost } \\
\text { - Service Level Agreements } \\
\text { [SLAs] \& service } \\
\text { degradation penalties } \\
\text { - Fault tolerance } \\
\text { - Services perform cost, load, } \\
\text { perform time, Reliability }\end{array}$ & \multirow{3}{*}{$\begin{array}{ll}\text { - } & \text { IoT Coverage } \\
\text { - } & \text { Availability } \\
\text { - } & \text { Response time } \\
\text { - } & \text { Security } \\
\text { - } & \text { Data Quality } \\
\text { - } & \text { Energy } \\
\text { - } & \text { Bandwidth } \\
\text { - } & \text { Consumption } \\
& \text { / Efficiency }\end{array}$} \\
\hline $\begin{array}{l}\text { Network } \\
\text { Layer }\end{array}$ & $\begin{array}{l}\text { - Latency, Bandwidth, Delay, } \\
\quad \text { Packet loss rate, Jitter } \\
\text { - Network resources utilization } \\
\text { - Life span of sensing networks } \\
\text { - } \begin{array}{l}\text { Reliability, } \\
\text { Real-time }\end{array} \\
\end{array}$ & \\
\hline $\begin{array}{l}\text { Perception } \\
\text { Layer }\end{array}$ & 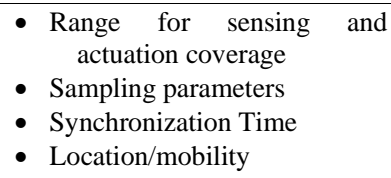 & \\
\hline
\end{tabular}

From the literature review some of the key QoS parameters at different layers are considered for distributable across layers and for adaptability. Some of QoS parameters of application layer are: service time, services availability, service delay, service accuracy, service load, service priority, information accuracy, network deployment cost, service usage cost, SLAs \& penalties for service degradation and fault tolerance and more.

The QoS parameters of network layer are: latency, bandwidth, packet loss rate, delay, jitter, and network resources utilization, life time of sensing networks, reliability, and throughput and real-time.

The QoS parameters for perception layer are: Range for Sensing and actuation coverage, sampling parameters, time synchronization and location/mobility. The QoS parameters common across layers are: IoT coverage, response time, energy consumption / efficiency.

The volume and variety of QoS parameters across layers demand the need for adaptability at each layer as adaptability and distribution is minimally addressed. The QoS parameters are measured and tracked by different SLAs defined as contractual agreements between users and service providers for providing QoS for any specific IoT implementation. The functional modules specific to layer(s) are non-adaptable and non-distributable. The functional modules would include functionalities like making the decisions, computations, analysis, interoperability between layers, control functions, data gathering and many more IoT functions and domain specific functions. The functional modules which are adaptable can be distributed / re-distributed across layers for effective QoS achievement. Let us consider the set of layers and functional modules for an IoT environment as $L$ and $M$.

$$
\text { Let } L=(1,2 \ldots i) \text { be the set of layers }
$$

For three layer architecture the value of $i=3$. The total number of layers i, varies based on the number of layers of the IoT system.

Let $M=(1,2,3 \ldots j)$ be the complete set of functional modules across layers (modules of all layers).

The total number of functional modules $\mathrm{j}$, varies based on the number of functional modules in each of the layer. Some ground rules to be considered for defining the functional modules:

- $\quad$ Each of the functional module is considered distinct by its name, function and action.

- If the name of the functional module is same it is considered to perform same actions / function irrespective of the layer.

For example 'compression' as a functional module is assumed to perform compression using same 'algorithm' or 'steps' irrespective of the layer it is implemented otherwise it is considered as different functional module. So if we consider it as same functional module which can either be implemented in layer 1 , then we represent the ordered pair as (1, 'compression') and as (3, 'compression') if it is implemented at layer 3.

Adaptability of functional modules may depend on two factors: (a) Depends on the external factors like data, traffic, and environment variables etc.

\section{Published By:}

Blue Eyes Intelligence Engineering 
and (b) dependency with functional modules of other layers for the overall functions of the IoT system. The proposed QoS framework defined here provides the solution by categorization \& optimization of functional modules in the layered architecture. The IoT functional modules can be distributable and can be adapted across layer(s) for effective QoS.

Layer specific (non-adaptable and non-distributable) functional modules: The functional modules which are layer specific, non-adaptable and non-distributable are defined by the intersection of the sets of functional modules.

For identifying and defining the distinct set of functional modules and type of functional modules as: (i) distributable / adaptable modules and (ii) layer specific, we apply the concepts of set theory. For example, let

$M_{1}=$ ('dashboard-control', 'compression', 'decision making', 'data sharing') - be functional modules of layer 1.

$M_{3}=$ ('decision making', 'compression', 'data sharing', 'sensing')- be the functional modules of layer 3.

$M_{1} \cap M_{3}=$ ('dashboard-control', 'sensing') is the set layer specific which are non-adaptable and non-distributable functional modules of layer 1 and 3 which will be defined as ordered pair as: $\{(1$, 'dashboard-control'), (3, 'sensing')\}.

The generic layer specific sets for each layer can be defined as:

$M_{1 S}=\left(m_{1 s 1}, m_{1 s 2}, m_{1 s 3} \ldots . . m_{1 s n}\right)$ modules specific for layer 1,

$M_{2 s}=\left(m_{2 s 1}, m_{2 s 2}, m_{2 s 3} \ldots . . m_{2 s n}\right)$ modules specific for layer 2,

$M_{3 S}=\left(m_{3 s 1}, m_{3 s 2}, m_{3 s 3} \ldots . . m_{3 s n}\right)$ modules specific for layer 3.

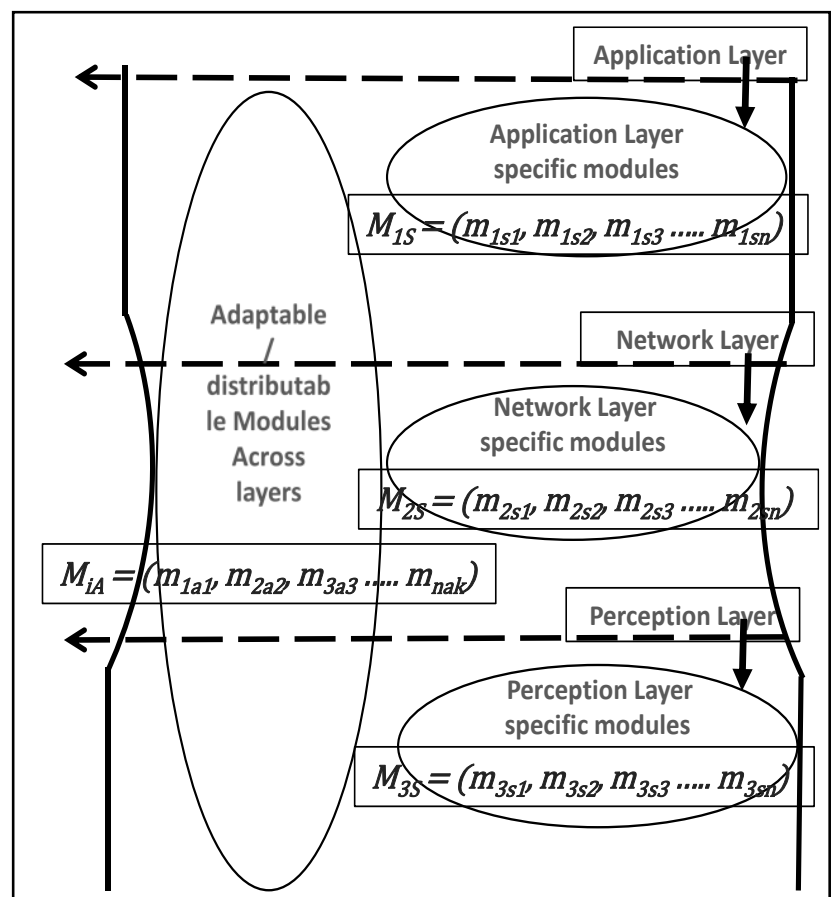

Fig. 2. Layer specific and adaptable functional modules in a three Layer hourglass IoT architecture.

Adaptable (adaptable and distributable) functional modules: The functional modules which are adaptable, adaptable and distributable are defined by the mutually exclusive set of sets of functional modules. For the defined sets $M_{1}$ and $M_{3}$ as:
$\left(M_{1} \cup M_{3}\right)-\left(M_{1} \cap M_{3}\right)=$ ('compression', 'decision making', 'data sharing') is the set of adaptable functional modules are can either be adapted at layer 1 or layer 3 . If we adapt both modules at layer 3 then the ordered pair will be defined as \{(3, 'compression'), (3, 'decision making'), (3, 'data sharing')\}.

The generic adaptable sets for each layer can be defined as:

$M_{i A}=\left(m_{1 a 1}, m_{2 a 2}, m_{3 a 3} \ldots . . m_{n a k}\right)$ adaptable modules across layers. Here $\mathrm{n}$ is the number of layers and adaptable modules vary from 1 to $\mathrm{k}$, and $\mathrm{k}$ is the total number of adaptable modules of all layers. A generic lathering of functional modules specific to layers and distributable modules are shown in Fig 2.

The set of functional modules $M_{i A}$ are layer specific functions which are non-distributable. When the functions are not similar then the functions will be mutually exclusive with respect to the functions of other layer, where distributiveness is minimal. Adaptability and distributiveness can be incorporated in such heterogeneous IoT environment by keeping the QoS specified or for improving the QoS.

\section{IMPLEMENTATION AND RESULTS}

Implementation: From our categorization and identification we consider the functional modules 'data redundancy' or 'data compression' at the perception layer as adaptable modules. It is illustrated that the bandwidth and latency improvement is achieved if we adapt the modules either to eliminate 'data redundancy' or 'data compression' at the perception layer. The illustration is done using the defined cross layer QoS architecture for IoT environment. The IoT implementation is done using a mobile APP (Android) for the functionalities of perception layer (for 'data gathering' and other functionalities) and application layer (for data processing and data access and more).

The application designed is implemented and run for a construction company for six months at different site locations to capture the field data of objects movements (material and labor movement data). The Fig. 3 shows the specific implementation block diagram.

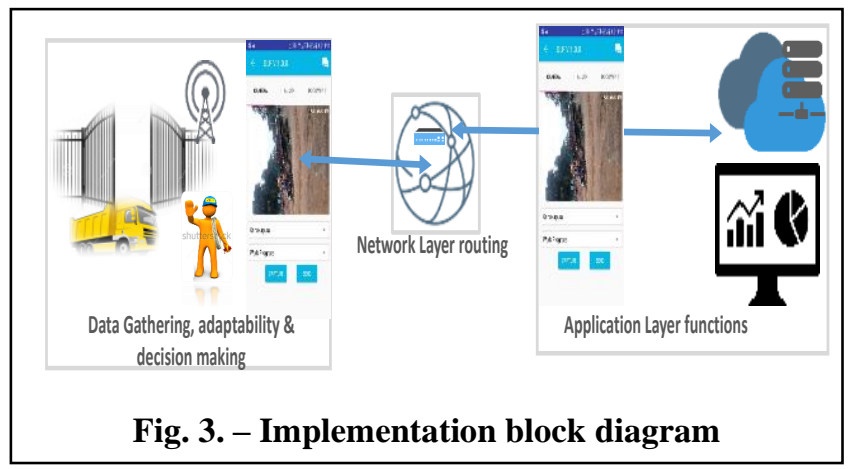

Verification: Implementation is verified for QoS parameters bandwidth, energy and latency. The bandwidth parameter is measured based on the users (number of applications) with different data rates for different types of data like text, images, audio, video and documents on different network scenarios like GSM, data rate package, exclusive IP network (WiFi) and some of $4 \mathrm{G}$ networks with devices of different capacities. 


\section{A Cross layer QoS Framework for Heterogeneous IoT Environment}

Technology Stack: Android, Java, MySQL for storing the data, cloud to host the mobile APP and web application developed using PHP.

Testing conditions and duration: The application is adapted and run for a construction company for a duration of six months and at situations with and without internet connections.

Implementation is carried out using the defined architecture and QoS framework to verify the adaptation, learning, and security aspects. The implemented android mobile application does the following functions/ functional modules categorized in perception and application layers as below:

- Capture data at fields using the GPS and time sensor. The data captured includes - images, documents, audio and sensed data for load/unload/arrival of materials. The captured data forms the inward inventory, crew data counts and machinery run hours and distances.

- The perception layer implements the adaptability 'data compression'.

- Local caching module (adaptable depending on network availability) take care of decision making.

- Application layer component pushes data to cloud storage. The data is shown in summary in the dashboard and same made available for data analytics.

- The security is implemented by providing access to the system by authorized login id and password.

Results: The system used functional modules for local adaption and decision making at perception layer and application layer for processing the gathered data. Verification is done for varied file sizes from $50 \mathrm{~kb}$ to $10 \mathrm{Mb}$ to find the latency time which directly related to bandwidth and energy. The adaptable module at perception layer compress the file sizes from the initial size (captured image size) of $50 \mathrm{~Kb}$ to $2 \mathrm{Mb}$. The data of standard files sizes, network speed and latency is shown in Table 2. The graph in Fig. 4 and Fig. 5 shows the file size and latency for varied file sizes in different network speed conditions pre-adaptation and post-adaptation.

From the results it is evident that the volume of data exchanged based on adaptability improves the energy and bandwidth (latency) efficiency. The graphs indicate the energy and bandwidth consumption before and after adaption. The results shows that the adaptability and optimization improves the energy conservation, computing power in turn improves the bandwidth efficiency and optimal storage leads to improvement of QoS in IoT environment.
TABLE 2 Latency for different file sizes / network speeds

\begin{tabular}{|c|c|c|c|c|}
\hline $\begin{array}{c}\text { File Size } \\
\text { Pre-adaption } \\
\text { (In Kb) }\end{array}$ & $\begin{array}{c}\text { File Size } \\
\text { Post-adaptio } \\
\text { n (In Kb) }\end{array}$ & $\begin{array}{l}\text { Network } \\
\text { Speed }\end{array}$ & $\begin{array}{l}\text { Time Taken } \\
\text { / Latency - } \\
\text { Pre-adaption } \\
\text { (standard } \\
\text { conditions) }\end{array}$ & $\begin{array}{c}\text { Time Taken } \\
\text { / Latency - } \\
\text { Post-adaptio } \\
\text { n (standard } \\
\text { conditions) }\end{array}$ \\
\hline \multirow{4}{*}{$\begin{array}{l}50 \\
50 \\
50 \\
50\end{array}$} & \multirow{4}{*}{$\begin{array}{c}21 \\
21.5 \\
20 \\
20\end{array}$} & 50 & 5 & 2 \\
\hline & & 50 & 0.4 & 0.16 \\
\hline & & 50 & 0.2 & 0.08 \\
\hline & & 50 & 0.1 & 0.04 \\
\hline \multirow{4}{*}{$\begin{array}{l}200 \\
200 \\
200 \\
200\end{array}$} & \multirow{4}{*}{$\begin{array}{l}80 \\
84 \\
86 \\
84\end{array}$} & 200 & 22 & 8.8 \\
\hline & & 200 & 3 & 1.2 \\
\hline & & 200 & 0.4 & 0.16 \\
\hline & & 200 & 0.1 & 0.04 \\
\hline \multirow{4}{*}{$\begin{array}{l}500 \\
500 \\
500 \\
500\end{array}$} & \multirow{4}{*}{$\begin{array}{l}205 \\
215 \\
215 \\
215\end{array}$} & 500 & 55 & 22 \\
\hline & & 500 & 8 & 3.2 \\
\hline & & 500 & 1 & 0.4 \\
\hline & & 500 & 0.2 & 0.08 \\
\hline \multirow{4}{*}{$\begin{array}{l}1000 \\
1000 \\
1000 \\
1000\end{array}$} & \multirow{4}{*}{$\begin{array}{l}400 \\
400 \\
410 \\
400\end{array}$} & 1000 & 52 & 20.8 \\
\hline & & 1000 & 17 & 6.8 \\
\hline & & 1000 & 3 & 1.2 \\
\hline & & 1000 & 1 & 0.4 \\
\hline \multirow{4}{*}{$\begin{array}{l}5000 \\
5000 \\
5000 \\
5000\end{array}$} & \multirow{4}{*}{$\begin{array}{l}2100 \\
2000 \\
2150 \\
2050\end{array}$} & 5000 & 563 & 225.2 \\
\hline & & 5000 & 88 & 35.2 \\
\hline & & 5000 & 16 & 6.4 \\
\hline & & 5000 & 5 & 2 \\
\hline \multirow{4}{*}{$\begin{array}{l}10000 \\
10000 \\
10000 \\
10000\end{array}$} & \multirow{4}{*}{$\begin{array}{l}4000 \\
4100 \\
4300 \\
4200\end{array}$} & 10000 & 1126 & 450.4 \\
\hline & & 10000 & 176 & 70.4 \\
\hline & & 10000 & 32 & 12.8 \\
\hline & & 10000 & 11 & 4.4 \\
\hline
\end{tabular}

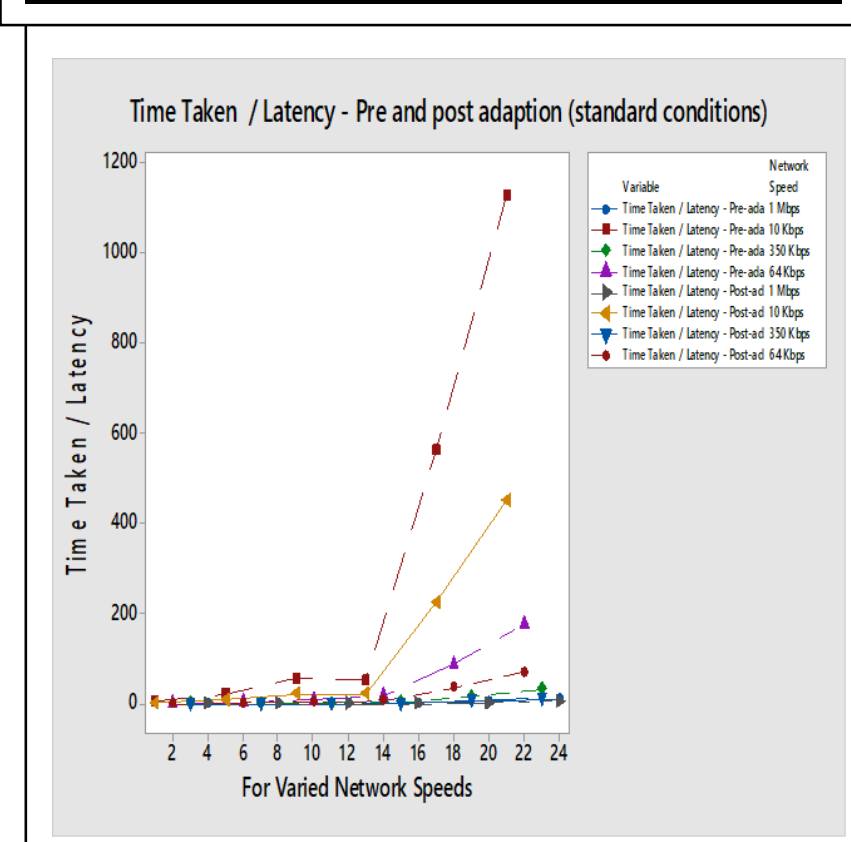

Fig. 5. - Latency pre and post adaption for varied network speeds 


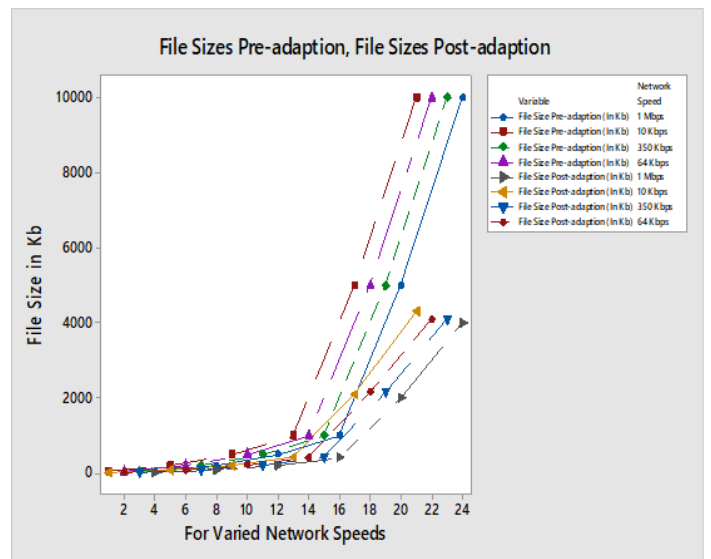

Fig. 4. - File sizes pre and post adaption for varied network speeds

\section{CONCLUSION AND FUTURE WORK}

The new QoS framework and the overall scope of research is identified and defined based on the investigations of various QoS implementations in IoT environment by different researchers. By the reviews it is understood that achieving

QoS by cross layer QoS model with decision making and adaptability is less addressed. The model categorizes the IoT functional modules for adaptability, distributable and decision making. The cross layer QoS framework is defined for achieving QoS optimization and improvement. The implementation of categorized modules at perception layer and application layer for energy efficiency and bandwidth optimizations show the trans-receiving and storage efficiencies. Implementations carried out using the proposed model to measure the QoS parameters bandwidth and latency before adaptation and post adaptation. The results show that the architecture, QoS framework and adaptable models help in improving the QoS parameter values and thereby improving the QoS in designed IoT system.

The future work will involve implementing machine learning and deep learning algorithms by varying adaptability in functional modules across layers to achieve effective QoS using defined architecture and framework.

\section{ACKNOWLEDGMENT}

Thanks to Jain University for providing access to resources and providing opportunity to work on research activities. Thanks to colleagues of Information Science and Engineering (ISE) department at MSRIT for support and ePrayag Software Private limited for facilitating the test environment and data for verifying the results.

\section{REFERENCES}

1. John A. Stankovic, Life Fellow, IEEE, "Research Directions for the Internet of Things", INTERNET OF THINGS JOURNAL, VOL. 1, NO. 1, FEBRUARY 2014.

2. J.Gubbi, R. Buyya, S. Marusic, and M. Palaniswami. "Internet of Things (IoT): A vision, architectural elements, and future directions." Future Generation Computer Systems, 2013.

3. Ling Li, Shancang Li, and Shanshan Zhao, "QoS-Aware Scheduling of Services-Oriented Internet of Things", IEEE TRANSACTIONS ON INDUSTRIAL INFORMATICS, VOL. 10, NO. 2, MAY 2014;

4. Saima Abdullah, Kun Yang, "A QoS Aware Message Scheduling Algorithm in Internet of Things Environment", 2013 IEEE Online Conference on Green Communications (OnlineGreenComm).

5. Tingxun SHI, Ruizhi WANG, Daolan ZHANG, Wenpin JIAO*, Bing XIE, "Quality Driven Design of Program Frameworks for Intelligent
Sensor Applications”, 2013 20th Asia-Pacific Software Engineering Conference

6. Ren Duan1,2, Xiaojiang Chen1, Tianzhang Xing1, "A QoS Architecture for IOT", 2011 IEEE International Conferences on Internet of Things, and Cyber, Physical and Social Computing

7. Medha Shah1 D.B.Kulkarni, "Enabling QoS Support for Multi-Core Message Broker in Publish/Subscribe System", Advance Computing Conference (IACC), 2014 IEEE International

8. Rafael Perazzo Barbosa Mota, Daniel M. Batista, "A RFID QoS Mechanism for IoT Tracking Applications", Wireless and Pervasive Computing (ISWPC), 2013 International Symposium on, IEEE 2012

9. Marie-Aurélie Nef, Leonidas Perlepes, Sophia, Karagiorgou, George I. Stamoulis, Panayotis K. Kikiras, "Enabling QoS in the Internet of Things", CTRQ 2012 : The Fifth International Conference on Communication Theory, Reliability, and Quality of Service

10. O. Vermesan et al., "Internet of Things Strategic Research Roadmap", European Research cluster on the Internet of Things, Cluster Strategic Research Agenda 2011.

11. Zhou Ming and Ma Yan, "A Modeling and Computational Method for QoS in IOT", Software Engineering and Service Science (ICSESS), 2012 IEEE 3rd International Conference

12. Jia-Ming Liang,Member, IEEE, Jen-Jee Chen,Member, IEEE, Hung-Hsin Cheng, and Yu-Chee Tseng, Fellow, IEEE, "An Energy-Efficient Sleep Scheduling With QoS Consideration in 3GPP LTE-Advanced Networks for Internet of Things", IEEE JOURNAL ON EMERGING AND SELECTED TOPICS IN CIRCUITS AND SYSTEMS, VOL. 3, NO. 1, MARCH 2013

13. Basic set https://www.math.uh.edu/ dlabate/settheory_Ashlock.pdf

theory:

14. Giuseppe Colistra, Virginia Pilloni, Luigi Atzori DIEE, "Objects that Agree on Task Frequency in the IoT: a Lifetime-Oriented Consensus Based Approach", University of Cagliari, Italy, 2014 IEEE World Forum on Internet of Things (WF-IoT)

15. Chien-Liang Fok, Christine Julien, The University of Texas at Austin Austin, Texas, USA, Gruia-Catalin Roman, Chenyang Lu Washington University in Saint Louis Saint Louis, MO, USA, "Challenges of Satisfying Multiple Stakeholders: Quality of Service in the Internet of Things", May 2112

16. Shancang Li, George Oikonomou, Theo Tryfonas, Thomas M. Chen, and Li Da Xu, "A Distributed Consensus Algorithm for Decision Making in Service-Oriented Internet of Things", IEEE TRANSACTIONS ON INDUSTRIAL INFORMATICS, VOL. 10, NO. 2, MAY 2014

17. 1524_D1.3_Architectural_Reference_Model_update v1.5, IoT-A, V1, 2012-07-16

18. Xianrong Zheng, Student Member, IEEE, Patrick Martin, Kathryn Brohman, and Li Da Xu, Senior Member, IEEE, "Cloud Service Negotiation in Internet of Things Environment A Mixed Approach", IEEE TRANSACTIONS ON INDUSTRIAL INFORMATICS, VOL. 10, NO. 2, MAY 2014

19. Ravi C Bhaddurgatte and Vijay Kumar B P, "A Review: QoS Architecture and Implementations in IoT environment", Research \& Reviews: Journal of Engineering and Technology, Sept 2015

\section{AUTHORS PROFILE}

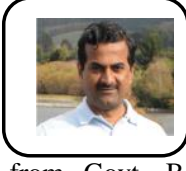

Mr. RAVI C BHADDURGATTE: received his M Tech degree in Computer science and Engineering from Manipal Institute of Technology (M I T), Manipal, Mangalore University with distinction in the year 1997, Bachelor's degree in Computer Science and Engineering from Govt. B. D. T. College of Engineering, Davanagere, Mysore University, India. He has 5 years of teaching experience and 22 years of experience in IT Services and currently working as Managing Director at ePrayag Software Pvt ltd. and pursuing research (Ph. D) at Jain University, Bangalore, India. His major areas of interest and research are Database systems, Business Intelligence and data warehousing, Big data and Analytics, Internet of Things (IoT), QoS in IoT, M2M communications, Cloud Computing, SDN and Mobile Computing. 


\section{A Cross layer QoS Framework for Heterogeneous IoT Environment}

Dr. VIJAYA KUMAR B. P. [SMIEEE] received his Ph. D degree from the Department of Electrical Communication Engineering, Indian Institute of Science (IISc), Bangalore in 2003, M. Tech degree in Computer Science and Technology from Indian Institute of Technology, IITR, with honours in 1992 and Bachelor's degree in Electronics and Communications from Mysore University with Distinction in the year 1987. He is currently heading the Department of Information Science and Engineering, M.S.Ramaiah Institute of Technology, Bangalore, Karnataka, where he is involved in research and teaching. His major areas of research are Computational Intelligence applications in Mobile, Ad-hoc and Sensor networks. He is having 26 years of Teaching and 20 years of Research experience. He has published around 65 papers which include journals, conferences and project proposals. His name is listed in Marquis Who's Who in the World, and Marquis Who's Who in Asia, 2012. He is a Senior Member of International Association of Computer Science and Information Technology (SMIACSIT), IEEE Senior Member and Life member of India Society for Technical Education (LMISTE).

Ms. KUSUMA S. M. [MIEEE, LMISTE] is working as an Assistant Professor in Telecommunication Engineering department, RIT. Completed her undergraduate from Mysore University in Electronics and Communication Eng., M. Tech in VLSI and Embedded systems from Visveswarayya Technological University, and presently pursuing her research work towards Sensors deployment in Embedded Sensor networks. Her areas of interests are Wireless Sensor Networks, Wireless Mobile Communication, Internet of Things, Embedded System Design, MIMO Technology, Machine learning and $4 \mathrm{~g} / 5 \mathrm{~g}$ Technology. 